Nouvelles perspectives en sciences sociales

Revue internationale de systémique complexe et d'études relationnelles

\title{
Y a-t-il une théorie en interdisciplinarité? Avant-propos
}

\section{Rachid Bagaoui et Alain Beaulieu}

Volume 7, numéro 1, octobre 2011

Sur le thème de l'interdisciplinarité

URI : https://id.erudit.org/iderudit/1007079ar

DOI : https://doi.org/10.7202/1007079ar

Aller au sommaire du numéro

Éditeur(s)

Prise de parole

ISSN

1712-8307 (imprimé)

1918-7475 (numérique)

Découvrir la revue

Citer ce document

Bagaoui, R. \& Beaulieu, A. (2011). Y a-t-il une théorie en interdisciplinarité?

Avant-propos. Nouvelles perspectives en sciences sociales, 7(1), 13-14.

https://doi.org/10.7202/1007079ar 


\title{
Y a-t-il une théorie en interdisciplinarité?
}

\section{Avant-propos}

\author{
RACHID BAgAOUI \\ Université Laurentienne, Sudbury \\ Alain Beaulieu \\ Université Laurentienne, Sudbury
}

e but initial de ce projet visait à répondre à la question «Y reçus ont préféré orienter la réflexion sur des thèmes connexes tels que la taxinomie du lexique relatif à l'interdisciplinarité, les études de cas ou encore la présentation de certains programmes interdisciplinaires. Cette hésitation à se confronter directement à la possibilité d'une théorie en interdisciplinarité peut être considérée comme une manière spécifique de répondre à la question : ou bien il n'y a tout simplement pas de théorie unifiée en interdisciplinarité, ou alors les études en interdisciplinarité n’ont pas atteint le degré de maturité nécessaire au développement d'une telle théorie.

Ce rapport ambigu et hésitant face à l'interdisciplinarité s'exprime aussi dans le processus d'évaluation des articles en tant que l'acceptation ou non des articles est en quelque sorte corrélée 
à l'ouverture ou à la fermeture des appréciateurs face à l'interdisciplinarité. Question théorique sans objet pour les uns, question fondamentale pour d'autres, les articles soumis n'ont pas eu ni le même accueil ni la même appréciation. Nous avons tenu compte dans la mesure du possible de tous les commentaires même ceux qui nous paraissaient quelque peu en marge du débat sur l'interdisciplinarité, convaincus de l'importance de leur contribution au débat sur l'interdisciplinarité.

Les textes proposés dans ce numéro spécial de la revue Nouvelles perspectives en sciences sociales abordent donc la question de l'interdisciplinarité sous différents angles qui permettent de poursuivre un dialogue ou d'initier de nouvelles discussions en proposant un certain nombre de réflexions fondamentales sur l'interdisciplinarité et en indiquant des domaines d'applications possibles. Il restera aux lecteurs actuels et à venir la tâche de déterminer si l'interdisciplinarité est destinée à n'être qu'un amalgame de pratiques et de notions hétérogènes issues des disciplines, si une théorie unifiée de l'interdisciplinarité peut être envisagée, ou encore s'il y a des savoirs proprement interdisciplinaires. La question initialement lancée demeure ainsi ouverte.

Les huit textes rassemblés dans ce numéro ont été rédigés par des auteurs provenant de cinq pays, ce qui illustre bien le caractère international des réflexions en interdisciplinarité. Nous tenons à remercier chaleureusement les auteurs ainsi que les appréciateurs qui ont contribué à la qualité de ce numéro spécial. Nous remercions également Valérie Bélanger qui a traduit le texte de Julie T. Klein ainsi que Simon Laflamme, directeur du programme de doctorat en sciences humaines de l'Université Laurentienne (Canada), pour son appui indéfectible en cours de route. Ce projet est une initiative du Centre international de recherche interdisciplinaire en sciences humaines (CIRISH) de l'Université Laurentienne (www.cirish.laurentienne.ca). 